\title{
Almost planar waves in anisotropic media
}

\author{
Mariana Haragus \\ Université de Franche-Comté \\ Département de Mathématiques \\ 16 route de Gray \\ 25030 Besançon cedex, France \\ Arnd Scheel \\ University of Minnesota \\ School of Mathematics \\ 206 Church St. S.E. \\ Minneapolis, MN 55455, USA
}

\begin{abstract}
We investigate corners and steps of interfaces in anisotropic systems. Starting from a stable planar front in a general reaction-diffusion-convection system, we show existence of almost planar interior and exterior corners. When the interface propagation is unstable in some directions, we show that small steps in the interface may persist. Our assumptions are based on physical properties of interfaces such as linear and nonlinear dispersion, rather than properties of the modeling equations such as variational or comparison principles. We also give geometric criteria based on the Cahn-Hoffman vector, that distinguish between the formation of interior and exterior corners.
\end{abstract}

Running head: Almost planar waves in anisotropic media

Corresponding author: Arnd Scheel, scheel@math.umn.edu

Keywords: corners in interfaces, anisotropy, Burgers equation, Cahn-Hoffmann vector 


\section{Introduction}

We study almost planar interface propagation in 2-dimensional, anisotropic, homogeneous, nonlinear media. The simplest example of front propagation is the scalar bistable reactiondiffusion equation

$$
u_{t}=\Delta_{x, y} u+u(1-u)(u-a),
$$

where $0<a<1$. Planar fronts connecting the stable homogeneous equilibria $u=0$ and $u=1$ may propagate in either direction $n=(\sin \varphi, \cos \varphi)$, depending upon the initial condition. In a recent article [8], we showed that almost planar fronts exist for $a \neq 1 / 2$ : the front position, traced for example by the level set $u^{-1}(1 / 2)$, is located on a curve that approaches two straight lines $y=\varepsilon|x|$ for $\varepsilon>0$ small, and the interface propagates in the direction of increasing $y$. Zooming out to a large spatial scale, the domain occupied by the 'winning' state that is left behind the front possesses an interior corner; see Figure 2.1, below.

Using comparison principles, the existence of such fronts can be established for large angles $\varepsilon$; see [3] for a related result. All these nonplanar fronts share the property that the asymptotic planar part of the front propagates towards the middle, center part of the interface. The results in [8] show that, at least for small angles $\varepsilon$, the existence and asymptotic stability of these interior corners is a universal feature in isotropic reaction-diffusion systems, $u \in \mathbb{R}^{N}$, that exhibit stable planar fronts. We point out that unstable, or pulsating, fronts may lead to different types of interfaces such as exterior corners, steps, or holes; see Figure 2.1. All these almost planar interfaces are referred to as corner defects.

Our focus here is on anisotropic media, where convection and diffusion do not respect the rotational symmetry of the plane. A simple example is given by

$$
u_{t}=\Delta_{x, y} u+u(1-u)(u-a)+\mu g(u, \nabla u),
$$

with $\mu$ small. The planar fronts that propagate in arbitrary directions for $\mu=0$ still exist for $\mu>0$ small, but the speed of propagation $c$ will typically depend on the direction, $c=c(\varphi)$. Our main results here establish the existence of almost planar interfaces as described above for general anisotropic reaction-diffusion systems. However, the direction of propagation and the angle at the corner relative to the direction of propagation now crucially depend on the function $c(\varphi)$. In particular, we show that the corners propagate in the tangential direction with speed $c^{\prime}(\varphi)$. Moreover, the domain occupied by the winning state behind the front may possess an exterior corner on the large spatial scale, as well.

Our work is motivated by a number of specific examples of front propagation in anisotropic media. As a first example, we cite models of cardio-vascular tissue [11]. Conductivity along fibers is larger by several orders of magnitude than across fibers. The electric potential can be 
modeled by a diffusion equation with a nonlinear source term and different diffusion coefficients for the directions across and along fibers, respectively. In one-domain models, these different diffusivities can be accounted for by a simple anisotropic rescaling. However, in two-domain models for intra- and extra-cellular tissue, the ratios of diffusivities typically differ and the resulting reaction-diffusion system is inherently anisotropic.

As a second example, we cite catalytic surface reaction, where diffusion rates of the reactants depend on the crystalline structure of the surface. Particularly well studied is the CO-oxidation on Pt-surfaces, where anisotropy may lead to breathing stripes and spatio-temporal chaos; [1]. We would also like to mention a slightly different approach to front propagation. The position of the front traces a curve in the plane, and on suitable spatio-temporal scales, the evolution of this curve is described by an eikonal equation; see, for example, [4] in the isotropic case and [2] in an anisotropic setting. All these results exploit comparison principles that are not available in our context of reaction-diffusion systems. Moreover, our results differ from these approaches in that we consider non-compact interfaces, which causes a number of technical difficulties due to essential spectrum in the linearized operators. Also, our almost planar interfaces are not smooth in the sharp interface limit: the asymptotic planar interfaces form an angle of contact at the center.

This paper is organized as follows. In Section 2, we describe our basic setup and hypotheses. We then state our main results on the existence of interior and exterior corners in Section 3. Section 4 contains the proofs of the theorems and we give some examples in Section 5 . We conclude with results in the case of lateral instabilities in Section 6 and a brief discussion.

Acknowledgments This work was partially supported by the MRT through grant ACI JC 1039 (M. H.) and by the NSF through grant DMS-0203301 (A. S.).

\section{Corner defects and hypotheses}

\section{Corner defects}

We consider the general reaction-diffusion system

$$
u_{t}=\nabla \cdot(a \nabla u)+f(u, \nabla u)+c(n \cdot \nabla) u,
$$

with $u=\left(u^{m}\right)_{1 \leq m \leq N} \in \mathbb{R}^{N}$ depending upon time $t$ and space $x=\left(x_{1}, x_{2}\right) \in \mathbb{R}^{2}$. Here $a=\left(a_{i j}^{m}\right)_{1 \leq i, j \leq 2}^{1 \leq m \leq N}$ such that

$$
(\nabla \cdot(a \nabla u))^{m}=a_{i j}^{m} \partial_{i j} u^{m},
$$

and $\sum_{i j} a_{i j}^{m} y_{i} y_{j} \geq M \sum_{i} y_{i}^{2}$, for some $M>0$ independent of $y=\left(y_{1}, y_{2}\right)$. The last term in (2.1) is induced by passing to a moving coordinate frame in the direction $n=(\sin \varphi, \cos \varphi) \in S^{1}$ 
with speed $c$, so that steady solutions to (2.1) are travelling waves propagating in the direction $n$ with speed $c$. The nonlinearity $f$ is assumed to be smooth. Obviously, the last term could be absorbed into the nonlinearity $f$ but we prefer to preserve the idea of a fixed (laboratory) reference frame for our considerations, in particularly when varying the speed $c$.

Throughout, we assume that for a given direction $n=n_{*}$ (or angle $\varphi=\varphi_{*}$ ) the system (2.1) possesses a planar traveling-wave solution connecting two homogeneous equilibria. More precisely, we make the following assumption.

Hypothesis 2.1 (Existence) We assume that there exist a direction $n=n_{*}$, a positive speed $c=c_{*} \geq 0$, and asymptotic states $q_{ \pm}$such that (2.1) possesses a smooth planar traveling-wave solution $u(t, x)=q_{*}\left(n_{*} \cdot x\right)$ connecting $q_{-}$and $q_{+}$, i.e.

$$
q_{*}(\zeta) \rightarrow q_{ \pm}, \quad \text { as } \quad \zeta \rightarrow \pm \infty
$$

The profile $q_{*}$ solves

$$
(a n \cdot n) u^{\prime \prime}+f\left(u, n u^{\prime}\right)+c u^{\prime}=0,
$$

with $n=n_{*}, c=c_{*}$, where' denotes differentiation with respect to $\zeta=n \cdot x$, the vector $($ an $\cdot n)$ is regarded as a diagonal $N \times N$-matrix, and $n u^{\prime}$ stands for the tensor product.

We are interested in almost planar interfaces. Denote by $\xi:=n^{\perp} \cdot x$ the direction perpendicular to the direction of propagation, in which $n^{\perp}=n^{\prime}(\varphi)=(\cos \varphi,-\sin \varphi)$. We recall below the definition and classification of corner defects.

Definition 2.2 (Corner defects) We call a solution $u$ to (2.1) an almost planar interface $\delta$-close to $q_{*}$, if $u$ is of the form

$$
u(x)=q_{*}(\zeta+h(\xi))+u_{1}(\xi, \zeta),
$$

with $h \in C^{2}(\mathbb{R})$ and

$$
\sup _{\xi \in \mathbb{R}}\left|h^{\prime}(\xi)\right|<\delta, \quad \sup _{\xi \in \mathbb{R}}\left\|u_{1}(\xi, \cdot)\right\|_{H^{1}\left(\mathbb{R}, \mathbb{R}^{N}\right)}<\delta, \quad\left|c-c_{*}\right|<\delta
$$

We say that $u$ is a planar interface if $h^{\prime \prime} \equiv 0$.

We call $u$ a corner defect if it is of the form (2.3), with $h^{\prime \prime} \not \equiv 0$, and $h^{\prime}(\xi) \rightarrow \eta_{ \pm}$as $\xi \rightarrow \pm \infty$. We say that

- $u$ is an interior corner if $\eta_{+}<\eta_{-}$;

- $u$ is an exterior corner if $\eta_{-}<\eta_{+}$; 
- $u$ is a step if $\eta_{+}=\eta_{-} \neq 0$;

- $u$ is a hole if $\eta_{+}=\eta_{-}=0$;

see Figure 2.1.

(a)

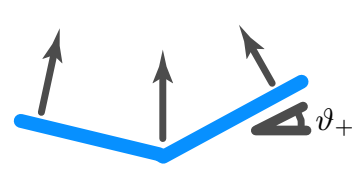

(b)

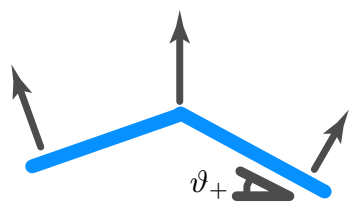

(c)

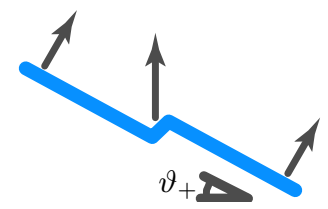

(d)

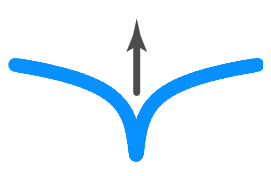

Figure 2.1: Schematic plot of the four different types of corner defects: interior corner (a), exterior corner (b), step (c), and hole (d). The middle arrows indicate the speed of the defect, whereas the left and right arrows indicate the normal speed of propagation of the asymptotic planar interfaces. The asymptotic slopes are $\eta_{ \pm}=\tan \vartheta_{ \pm}$.

\section{Linear transport}

We linearize the system (2.1) at the solution $q_{*}$ and obtain the linearized operator

$$
\mathcal{L}_{*} u=\nabla \cdot(a \nabla u)+f_{1}^{*} u+f_{2}^{*} \nabla u+c_{*}\left(n_{*} \cdot \nabla\right) u,
$$

where

$$
f_{1}^{*}=\partial_{1} f\left(q_{*}, n_{*} q_{*}^{\prime}\right), \quad f_{2}^{*}=\partial_{2} f\left(q_{*}, n_{*} q_{*}^{\prime}\right) .
$$

With the Fourier decomposition into transverse wavenumbers $k, u=v\left(n_{*} \cdot x\right) \mathrm{e}^{\mathrm{i} k\left(n_{*}^{\perp} \cdot x\right)}$, we find $\mathcal{L}(k) v=\left(a n_{*} \cdot n_{*}\right) v^{\prime \prime}+\mathrm{i} k\left(\left(a+a^{T}\right) n_{*} \cdot n_{*}^{\perp}\right) v^{\prime}-k^{2}\left(a n_{*}^{\perp} \cdot n_{*}^{\perp}\right) v+f_{1}^{*} v+f_{2}^{*}\left(n_{*} v^{\prime}+\mathrm{i} k n_{*}^{\perp} v\right)+c_{*} v^{\prime}$.

At $k=0$, we recover the one-dimensional linearized problem

$$
\mathcal{L}_{0} v:=\left(a n_{*} \cdot n_{*}\right) v^{\prime \prime}+f_{1}^{*} v+f_{2}^{*}\left(n_{*} v^{\prime}\right)+c_{*} v^{\prime}
$$

The following stability hypothesis reflects the typical case of fronts that are asymptotically stable in one space-dimension and also stable with respect to transverse perturbations in two space-dimensions. It is the essential ingredient to our construction of interior and exterior corners in Section 3.

Hypothesis 2.3 (Stability) We assume that the spectrum of $\mathcal{L}_{0}$ is contained in $\{\lambda \in \mathbb{C}$ : $\operatorname{Re} \lambda<0\} \cup\{0\}$, and the spectrum of $\mathcal{L}(k), k \neq 0$, is contained in $\{\lambda \in \mathbb{C}: \operatorname{Re} \lambda<0\}$. Moreover, we assume that $\mathcal{L}_{0}$ is Fredholm with index zero, has a one-dimensional generalized kernel spanned by $q_{*}^{\prime}$, and that the algebraically simple eigenvalue $\lambda=0$ of $\mathcal{L}_{0}$ continues to an eigenvalue $\lambda(k)$ to $\mathcal{L}(k)$, for small $k$, with $\lambda^{\prime \prime}(0)<0$. 
Notice the symmetry $\lambda \mapsto \bar{\lambda}, k \mapsto-k$, which implies that $\operatorname{Re} \lambda$ and $\operatorname{Im} \lambda$ are even and odd in $k$, respectively. In particular, the even, respectively odd, order derivatives of $\lambda$ at $k=0$ are real, respectively purely imaginary, such that the stability assumption is merely a sign condition on $\lambda^{\prime \prime}(0)$.

We set $e_{0}=q_{*}^{\prime}$, which typically belongs to the one-dimensional kernel of $\mathcal{L}_{0}$ due to the translation invariance in $\zeta$. We denote by $e_{0}^{\text {ad }}$ the unique vector in the kernel of the adjoint $\mathcal{L}_{0}^{\text {ad }}$ normalized through $\left(e_{0}, e_{0}^{\text {ad }}\right)=1$, and by $e(k)$ the unique family of eigenvectors to the eigenvalue $\lambda(k)$ of $\mathcal{L}(k)$ obtained by normalizing $\left(e_{0}^{\text {ad }}, e(k)\right)=1$. (We use the notation $(\cdot, \cdot)$ for the scalar product in $L^{2}(\mathbb{R})$.) Differentiating the eigenvalue problem

$$
\mathcal{L}(k) e(k)=\lambda(k) e(k)
$$

with respect to $k$ at $k=0$, then taking the scalar product with $e_{0}^{\text {ad }}$ and exploiting that $e_{0}^{\text {ad }}$ is perpendicular to the image of $\mathcal{L}_{0}$, we find

$$
\lambda^{\prime}(0)=\left(\mathcal{L}^{\prime}(0) e_{0}, e_{0}^{\mathrm{ad}}\right)=\mathrm{i}\left(\left(\left(a+a^{T}\right) n_{*} \cdot n_{*}^{\perp}\right) e_{0}^{\prime}+f_{2}^{*}\left(n_{*}^{\perp} e_{0}\right), e_{0}^{\mathrm{ad}}\right) \in \mathrm{i} \mathbb{R}
$$

We call $c_{\mathrm{g}}:=\mathrm{i} \lambda^{\prime}(0)$ the (linear) group velocity.

\section{Nonlinear transport}

We now focus on the traveling-wave equation (2.2), which we consider with parameters $n$ and $c$. We are especially interested in the dependence of $c$ on $n$ (or the angle $\varphi$ that determines $n$ ). In order to find traveling waves for nearby directions of propagation $n \sim n_{*}$, we have to solve the one-dimensional system

$$
T(u ; c, n):=(a n \cdot n) u^{\prime \prime}+f\left(u, n u^{\prime}\right)+c u^{\prime}=0,
$$

near $u=q_{*}, c=c_{*}$, and $n=n_{*}$. Since $\partial_{c} T\left(q_{*} ; c_{*}, n_{*}\right)=q_{*}^{\prime}=e_{0}$ does not lie in the range of $\mathcal{L}_{0}$, the linearization with respect to $u$ and $c$ is onto and we may solve for $c=c(\varphi)$ and $u=u(\varphi)$. More precisely, we have the following result.

Lemma 2.4 Assume that Hypotheses 2.1 and 2.3 hold. Then there exists a positive constant $\varepsilon$, such that for each angle $\varphi$ with $\left|\varphi-\varphi_{*}\right|<\varepsilon$, the one-dimensional system (2.5) possesses a traveling-wave solution $q(\varphi)$ connecting $q_{-}$and $q_{+}$, and which propagates with speed $c=c(\varphi)$ in the direction $n=n(\varphi)$. Moreover, this traveling-wave satisfies Hypothesis 2.3 on stability, such that $\lambda(0 ; \varphi)=0$ and $\lambda^{\prime \prime}(0 ; \varphi)<0^{1}$.

We call $c=c(\varphi)$ the nonlinear dispersion relation.

\footnotetext{
${ }^{1}$ For the $\varphi$-dependent linear dispersion $\lambda(k ; \varphi)$ we shall always use ${ }^{\prime}$ to denote the partial derivative with respect $k$.
} 
We point out that linear and nonlinear dispersion are related at linear level. Indeed, differentiating (2.5) with respect to $\varphi$, substituting $n^{\prime}(\varphi)=n^{\perp}(\varphi)$, and taking the scalar product with $e_{0}^{\text {ad }}$, we find that

$$
c^{\prime}(\varphi)=\mathrm{i} \lambda^{\prime}(0 ; \varphi)=c_{\mathrm{g}}(\varphi) .
$$

In particular, the derivative $c^{\prime}(\varphi)$ is precisely the (linear) group velocity.

\section{Directional dispersion}

The nonlinear dispersion $c=c(\varphi)$ gives the speed of propagation in the direction $n(\varphi)$. We also consider the speed of propagation in the direction $n=n_{*}$ of the primary front, and therefore introduce the directional dispersion

$$
d(\varphi)=\frac{c(\varphi)}{\cos \left(\varphi-\varphi_{*}\right)} .
$$

Notice that in the case of isotropic media, $c(\varphi)=c_{*}$, and the traveling waves $q(\varphi)=q_{*}$ are simply obtained by rotating the primary front. In that case the quadratic term of $d$ appeared as the flux function in a Burgers transport equation after a suitable formal asymptotic expansion. We therefore sometimes refer to $d$ as the flux. Moreover, we say that

- $d$ is convex if $d^{\prime \prime}>0$;

- $d$ is concave if $d^{\prime \prime}<0$;

- $d$ is flat if $d^{\prime \prime} \equiv 0$;

for angles $\varphi$ in a neighborhood of $\varphi_{*}$.

\section{Stable interior and exterior corners}

We present our first results on existence, non-existence, and stability of interior and exterior corners.

\section{Existence}

We first state the results on existence and non-existence of interior and exterior corners. The proof of the following theorem is given in Section 4.

Theorem 1 (Existence of corner defects) We assume existence of a planar interface $q_{*}$ propagating in the direction $n_{*}$ with speed $c_{*}$, Hypothesis 2.1, that satisfies the stability assumption, Hypothesis 2.3. Then there exists a positive constant $\delta>0$ such that the following hold. 
If $c_{\mathrm{g}} \neq 0$, there are no almost planar traveling waves propagating in the direction $n_{*}$ with speed c close to $c_{*},\left|c-c_{*}\right|<\delta$.

If $c_{\mathrm{g}}=0$, we have the following three cases.

- If the flux $d$ is convex, then for each speed $c>c_{*},\left|c-c_{*}\right|<\delta$, there exists an interior corner defect propagating in the direction $n_{*}$ which is unique up to translation in $x$. For $c<c_{*},\left|c-c_{*}\right|<\delta$, there are no almost planar traveling waves.

- If the flux $d$ is concave, then for each speed $c<c_{*},\left|c-c_{*}\right|<\delta$, there exists an exterior corner defect propagating in the direction $n_{*}$ which is unique up to translation in $x$. For $c>c_{*},\left|c-c_{*}\right|<\delta$, there are no almost planar traveling waves.

- If the flux $d$ is flat, there are no almost planar traveling waves propagating in the direction $n_{*}$, for any speed $c$ with $\left|c-c_{*}\right|<\delta$.

Moreover, the corner defects above are to leading order given by

$$
q(x)=q_{*}(\zeta+h(\xi))+\mathrm{O}\left(\left|c-c_{*}\right|\right)
$$

in which $\zeta=n_{*} \cdot x, \xi=n_{*}^{\perp} \cdot x$, and the derivative of $h$ satisfies

$$
h^{\prime}(\xi)=\frac{\lambda^{\prime \prime}(0)}{d^{\prime \prime}\left(\varphi_{*}\right)} \beta \tanh (\beta \xi)+\mathrm{O}\left(\left|c-c_{*}\right| \mathrm{e}^{-2|\beta \xi|}\right), \quad \beta=\frac{\sqrt{2\left(c-c_{*}\right) d^{\prime \prime}\left(\varphi_{*}\right)}}{\lambda^{\prime \prime}(0)}<0 .
$$

The corner defects that we construct in this theorem are by definition asymptotically planar. More precisely, at $\xi= \pm \infty$ we find the planar interfaces

$$
q_{ \pm}\left(\zeta+\eta_{ \pm} \xi\right)=q_{*}\left(\zeta+\eta_{ \pm} \xi\right)+\mathrm{O}\left(\left|c-c_{*}\right|\right)
$$

in which $\eta_{ \pm}=\lim _{\xi \rightarrow \pm \infty} h^{\prime}(\xi)$. The normal directions to these interfaces $n_{ \pm}=\left(\sin \varphi_{ \pm}, \cos \varphi_{ \pm}\right)$ are obtained from the equality $\tan \left(\varphi_{ \pm}-\varphi_{*}\right)=\eta_{ \pm}$, and their normal propagation speed from the nonlinear dispersion relation $c_{ \pm}=c\left(\varphi_{ \pm}\right)$. Since both interfaces propagate with speed $c$ in the direction $n_{*}$ of the primary interface, we can directly calculate the angles $\varphi_{ \pm}$from the directional dispersion,

$$
c=d\left(\varphi_{ \pm}\right)
$$

Now, if the flux $d$ is convex, then necessarily $c>c_{*}$, and there are precisely two planar interfaces with angles $\varphi_{+}-\varphi_{*} \sim-\left(\varphi_{-}-\varphi_{*}\right)$, for each speed $c$ close to $c_{*}$. If the flux $d$ is concave, then $c<c_{*}$, and there are again two planar interfaces with angles $\varphi_{+}-\varphi_{*} \sim-\left(\varphi_{-}-\varphi_{*}\right)$, for each speed $c$ close to $c_{*}$. Finally, if the flux $d$ is flat, we have $d(\varphi)=c_{*}$, for angles close to $\varphi_{*}$, so that all planar interfaces move with the same speed $c_{*}$ in the direction $n_{*}$. In particular, 
this shows that asymptotically planar corner defects can only exist in the first two cases, and that they propagate in the direction $n_{*}$ with speed $c=d\left(\varphi_{ \pm}\right)$.

\section{Corner defects in case of non-zero group velocities}

The condition on the group velocity $c_{\mathrm{g}}$ in Theorem 1 can be interpreted in a slightly different fashion. We can pass in the system (2.1) to a comoving frame $x \mapsto x-c_{\mathrm{g}} n_{*}^{\perp} t$, which then adds a drift term $c_{\mathrm{g}}\left(n_{*}^{\perp} \cdot \nabla\right) u$ to the nonlinearity $f$. With this new nonlinearity, a straightforward computation shows that $\widetilde{c}_{\mathrm{g}}=0$. The nonlinear dispersion relation is easily obtained from the speed of propagation in the normal direction, corrected by $-c_{\mathrm{g}} \sin \left(\varphi-\varphi_{*}\right)$, such that

$$
\widetilde{d}(\varphi)=\frac{c(\varphi)-c_{\mathrm{g}} \sin \left(\varphi-\varphi_{*}\right)}{\cos \left(\varphi-\varphi_{*}\right)}=d(\varphi)-c_{\mathrm{g}} \tan \left(\varphi-\varphi_{*}\right)
$$

and, in particular, $\widetilde{d}^{\prime \prime}\left(\varphi_{*}\right)=d^{\prime \prime}\left(\varphi_{*}\right)$.

Consequently, interior and exterior corners "always" exist, for convex and concave flux, respectively, but drift along the interface with speed approximately given by the group velocity $c_{\mathrm{g}}$ : the drift speed for small linear perturbations actually determines the drift of nonlinear, corner-like defects along the interface.

We point out that the third possibility, $d^{\prime \prime} \equiv 0$ has to be checked in the frame moving with the group velocity along the interface, that is it has to be checked for the modified flux $\widetilde{d}$.

Remark 3.1 In the context of material science, propagation of interfaces in anisotropic media has been studied in the sharp interface limit. To leading order, the evolution of the interface is governed by an eikonal equation, where the direction of propagation of points on the interface is given by the so-called Cahn-Hoffmann vector [5, 6]. In our context, the Cahn-Hoffmann vector is given by the sum of tangential group velocity and normal speed of propagation, $c_{g} n^{\perp}+c n$. Our results show that indeed small defects in interfaces propagate in the direction of the CahnHoffmann vector.

\section{Stability}

The corner defects found in Theorem 1 have the same stability properties as the interior corners constructed in the isotropic case in [8, Theorems 2 and 3]. The results in [8] can be easily extended to the present situation and we therefore refer to [8] for the precise statements of these results and for the proofs. Roughly speaking, they show that the interior and exterior corners in Theorem 1 are asymptotically stable with respect to perturbations which are exponentially localized in the direction perpendicular to the direction of propagation.

The first result asserts asymptotic stability for fully localized perturbations

$$
v(x)=\cosh (\delta \xi)^{-1} w(\xi, \zeta), \quad w \in H^{2}\left(\mathbb{R}^{2}, \mathbb{R}^{N}\right),
$$


in which $\delta$ is chosen sufficiently small, $\delta=\mathrm{O}\left(\left|c-c_{*}\right|^{1 / 2}\right)$; [8, Theorem 2]. The second result gives asymptotic stability with asymptotic phase for a class of non-localized perturbations which allow for changing the position of the corner. These perturbations are localized in any spatial direction except along the corner interface; [8, Theorem 3].

The proofs rely upon a careful analysis of the spectrum of the linearization about the corner defect. The spectrum is strictly contained in the left complex half-plane, for fully localized perturbations, with an additional geometrically double eigenvalue at the origin, for the nonlocalized perturbations. The eigenvectors associated to this eigenvalue are the derivatives with respect to $\xi$ and $\zeta$ of the corner defect, and therefore allow for changing the position of the corner. Nonlinear stability is then obtained by a standard fixed point argument.

\section{Spatial dynamics - existence of corners}

We give here the proof of Theorem 1. We mainly follow the proof from the isotropic case given in [8], and will therefore refer to [8] for arguments which remain unchanged.

Proof. [of Theorem 1]

We start by rewriting the stationary equation to (2.1) with $n=n_{*}$ as a dynamical system in which the time-like variable is $\xi=n_{*}^{\perp} \cdot x$, the direction perpendicular to the direction of propagation of the planar front. In the coordinates $(\xi, \zeta)=\left(n_{*}^{\perp} \cdot x, n_{*} \cdot x\right)$ we find

$$
\left(a n_{*}^{\perp} \cdot n_{*}^{\perp}\right) u_{\xi \xi}+\left(\left(a+a^{T}\right) n_{*} \cdot n_{*}^{\perp}\right) u_{\xi \zeta}+\left(a n_{*} \cdot n_{*}\right) u_{\zeta \zeta}+f\left(u, n_{*} u_{\zeta}+n_{*}^{\perp} u_{\xi}\right)+c u_{\zeta}=0 .
$$

We set $v=u_{\xi}, \mathbf{u}=(u, v)^{T}$, and obtain the first-order system

$$
\mathbf{u}_{\xi}=\mathcal{A}(c) \mathbf{u}+\mathcal{F}(\mathbf{u})
$$

where

$$
\mathcal{A}(c)=\left(\begin{array}{cc}
0 & \text { id } \\
-\left(a n_{*}^{\perp} \cdot n_{*}^{\perp}\right)^{-1}\left(\left(a n_{*} \cdot n_{*}\right) \partial_{\zeta \zeta}+c \partial_{\zeta}\right) & -\left(a n_{*}^{\perp} \cdot n_{*}^{\perp}\right)^{-1}\left(\left(a+a^{T}\right) n_{*} \cdot n_{*}^{\perp}\right) \partial_{\zeta}
\end{array}\right),
$$

and

$$
\mathcal{F}(\mathbf{u})=\left(\begin{array}{c}
0 \\
-\left(a n_{*}^{\perp} \cdot n_{*}^{\perp}\right)^{-1} f\left(u, n_{*} u_{\zeta}+n_{*}^{\perp} v\right)
\end{array}\right) .
$$

At $c=c_{*}$ the system has a line of equilibria obtained from the primary planar front $u=q_{*}(\cdot)$ together with the translations $q_{*}(\cdot+h)$,

$$
\mathbf{q}_{*}^{h}=\left(\begin{array}{c}
q_{*}(\cdot+h) \\
0
\end{array}\right)
$$


The linearization of (4.1) about $\mathbf{q}_{*}^{0}$ is given by the operator

$$
\mathcal{A}_{*}=\left(\begin{array}{cc}
0 & \text { id } \\
2 \mathcal{L}^{\prime \prime}(0)^{-1} \mathcal{L}_{0} & -2 \mathcal{L}^{\prime \prime}(0)^{-1}\left(\mathrm{i} \mathcal{L}^{\prime}(0)\right)
\end{array}\right)
$$

in which $\mathcal{L}_{0}$ is the linear operator defined in $(2.4), \mathcal{L}^{\prime}(0)$ represents the derivative with respect to $k$ of $\mathcal{L}(k)$ at $k=0$,

$$
\mathrm{i} \mathcal{L}^{\prime}(0) v=-\left(\left(a+a^{T}\right) n_{*} \cdot n_{*}^{\perp}\right) v^{\prime}-f_{2}^{*}\left(n_{*}^{\perp} v\right)
$$

and $\mathcal{L}^{\prime \prime}(0)$ the second derivative given by

$$
\mathcal{L}^{\prime \prime}(0)=-2\left(a n_{*}^{\perp} \cdot n_{*}^{\perp}\right) .
$$

We consider $\mathcal{A}_{*}$ as a closed linear operator in $Y=\left(H^{1} \times L^{2}\right)\left(\mathbb{R}, \mathbb{R}^{N}\right)$ with domain of definition $Y^{1}=\left(H^{2} \times H^{1}\right)\left(\mathbb{R}, \mathbb{R}^{N}\right)$. Hypothesis 2.3 implies the following result on the spectrum of $\mathcal{A}_{*}$.

Lemma 4.1 Assume that Hypothesis 2.3 holds. Then the spectrum of $\mathcal{A}_{*}$ satisfies

$$
\operatorname{spec}\left(\mathcal{A}_{*}\right) \cap\{\nu \in \mathbb{C}:|\operatorname{Re} \nu| \leq \epsilon\}=\{0\},
$$

for some $\epsilon>0$.

If $c_{\mathrm{g}} \neq 0$, the eigenvalue 0 is simple.

If $c_{\mathrm{g}}=0$, the eigenvalue 0 is algebraically double and geometrically simple.

Proof. For $\nu \in \mathbb{C}$ we have to solve

$$
\left(\mathcal{A}_{*}-\nu \mathrm{id}\right) \mathbf{u}=\mathbf{f}
$$

Set $\mathbf{u}=(u, v), \mathbf{f}=(f, g)$, and find the equivalent system

$$
\begin{aligned}
v-\nu u & =f \\
2 \mathcal{L}^{\prime \prime}(0)^{-1}\left(\mathcal{L}_{0} u-\mathrm{i} \mathcal{L}^{\prime}(0) v\right)-\nu v & =g
\end{aligned}
$$

so that (4.3) has for $\mathbf{f} \in Y$ a unique solution $\mathbf{u} \in Y^{1}$ provided the equation

$$
\mathcal{L}_{0} u-\mathrm{i} \nu \mathcal{L}^{\prime}(0) u-\frac{\nu^{2}}{2} \mathcal{L}^{\prime \prime}(0) u=\tilde{f}:=\frac{1}{2} \mathcal{L}^{\prime \prime}(0)(g+\nu f)+\mathrm{i} \mathcal{L}^{\prime}(0) f,
$$

has a unique solution $u \in H^{2}\left(\mathbb{R}, \mathbb{R}^{N}\right)$. At $\nu=\mathrm{i} k$ we find

$$
\mathcal{L}(k) u=\tilde{f},
$$

so that the first part of the lemma follows from Hypothesis 2.3 on stability. 
Next, zero is a geometrically simple eigenvalue of $\mathcal{A}_{*}$ with eigenvector $\mathbf{e}_{0}=\left(e_{0}, 0\right)=\left(q_{*}^{\prime}, 0\right)$. A principal eigenvector $\mathbf{e}_{1}=\left(u_{1}, v_{1}\right)$ is obtained from

$$
v_{1}=e_{0}, \quad \mathcal{L}_{0} u_{1}=\mathrm{i} \mathcal{L}^{\prime}(0) e_{0}
$$

The solvability condition for the second equation,

$$
\left(\mathrm{i} \mathcal{L}^{\prime}(0) e_{0}, e_{0}^{\mathrm{ad}}\right)=0
$$

is precisely the condition on the group velocity $c_{\mathrm{g}}=0$. Consequently, zero is algebraically simple if $c_{\mathrm{g}} \neq 0$, and at least algebraically double otherwise. Finally, the condition on the second derivative $\lambda^{\prime \prime}(0)<0$ implies that if $c_{\mathrm{g}}=0$, the algebraic multiplicity of zero is precisely two, and the lemma is proved.

Now, we go back to the proof of Theorem 1. Assume first that $c_{\mathrm{g}}=0$. Then the eigenvalue of $\mathcal{A}_{*}$ in the origin is algebraically double with kernel and generalized kernel spanned by

$$
\operatorname{ker} \mathcal{A}_{*}=\operatorname{span}\left(\mathbf{e}_{0}\right), \mathbf{e}_{0}=\left(\begin{array}{c}
e_{0} \\
0
\end{array}\right), \quad \operatorname{gker} \mathcal{A}_{*}=\operatorname{span}\left(\mathbf{e}_{0}, \mathbf{e}_{1}\right), \mathbf{e}_{1}=\left(\begin{array}{c}
-\mathrm{i} e^{\prime}(0) \\
e_{0}
\end{array}\right),
$$

with $\mathcal{A}_{*} \mathbf{e}_{1}=\mathbf{e}_{0}$. Here $e^{\prime}(0)$ is the derivative with respect to $k$ at $k=0$ of the eigenvectors $e(k)$ to $\mathcal{L}(k)$; see Section 2.

We construct the spectral projection onto the generalized kernel, with the help of the $L^{2} \times L^{2}$ adjoint

$$
\mathcal{A}_{*}^{\mathrm{ad}}=\left(\begin{array}{cc}
0 & 2 \mathcal{L}_{0}^{\mathrm{ad}} \mathcal{L}^{\prime \prime}(0)^{-1} \\
\mathrm{id} & -2\left(\mathrm{i} \mathcal{L}^{\prime}(0)\right)^{\mathrm{ad}} \mathcal{L}^{\prime \prime}(0)^{-1}
\end{array}\right),
$$

where $\mathcal{L}_{0}^{\text {ad }}$ and $\mathcal{L}^{\prime}(0)^{\text {ad }}$ are the $L^{2}$-adjoints of $\mathcal{L}_{0}$ and $\mathcal{L}^{\prime}(0)$, respectively. The kernel and generalized kernel of $\mathcal{A}_{*}^{\text {ad }}$ are given by

$$
\operatorname{ker} \mathcal{A}_{*}^{\mathrm{ad}}=\operatorname{span}\left(\mathbf{e}_{0}^{\mathrm{ad}}\right), \mathbf{e}_{0}^{\mathrm{ad}}=\frac{1}{\lambda^{\prime \prime}(0)}\left(\begin{array}{c}
2\left(\mathrm{i} \mathcal{L}^{\prime}(0)\right)^{\mathrm{ad}} e_{0}^{\mathrm{ad}} \\
\mathcal{L}^{\prime \prime}(0) e_{0}^{\mathrm{ad}}
\end{array}\right), \quad \operatorname{gker} \mathcal{A}_{*}^{\mathrm{ad}}=\operatorname{span}\left(\mathbf{e}_{0}^{\mathrm{ad}}, \mathbf{e}_{1}^{\mathrm{ad}}\right),
$$

with $\mathcal{A}_{*}^{\text {ad }} \mathbf{e}_{1}^{\text {ad }}=\mathbf{e}_{0}^{\text {ad }}$ and $\mathcal{L}_{0}^{\text {ad }} e_{0}^{\text {ad }}=0$. Here the adjoint eigenvectors are normalized such that

$$
\left\langle\mathbf{e}_{j}, \mathbf{e}_{j}^{\mathrm{ad}}\right\rangle_{L^{2} \times L^{2}}=0, \quad\left\langle\mathbf{e}_{1-j}, \mathbf{e}_{j}^{\mathrm{ad}}\right\rangle_{L^{2} \times L^{2}}=1, \quad j=0,1
$$

by taking $\left(e_{0}, e_{0}^{\text {ad }}\right)=1$. Notice that the principal vector $\mathbf{e}_{1}^{\text {ad }}$ can be computed explicitly, as well, but we do not need this result for the arguments below. The projection on the generalized kernel is then given as a bounded operator on $Y$ through

$$
P: Y \rightarrow Y, \quad P \mathbf{u}=\left\langle\mathbf{u}, \mathbf{e}_{1}^{\mathrm{ad}}\right\rangle_{L^{2} \times L^{2}} \mathbf{e}_{0}+\left\langle\mathbf{u}, \mathbf{e}_{0}^{\mathrm{ad}}\right\rangle_{L^{2} \times L^{2}} \mathbf{e}_{1}
$$


Similarly, to the shifted equilibria $\mathbf{q}_{*}^{h}$ we introduce the shifted linear operator $\mathcal{A}_{*}^{h}$, the shifted eigenvector $\mathbf{e}_{0}^{h}=\left(\left(q_{*}^{\prime}\right)^{h}(\cdot), 0\right)$, and, analogously, $\mathbf{e}_{1}^{h}, \mathcal{A}_{*}^{\mathrm{ad}, h}, \mathbf{e}_{j}^{\mathrm{ad}, h}, P^{h}$.

We now decompose

$$
\mathbf{u}=\mathbf{q}_{*}^{h}+\eta \mathbf{e}_{1}^{h}+\mathbf{w}^{h}, \text { with } P^{h} \mathbf{w}^{h}=P \mathbf{w}=0 .
$$

Here $h$ and $\eta$ are real functions depending upon $\xi$. This provides us with coordinates in a neighborhood of the family of equilibria $\mathbf{q}_{*}^{h}$. Substituting (4.5) into (4.1), we find

$$
\begin{aligned}
h_{\xi} \mathbf{e}_{0}^{h}+\eta_{\xi} \mathbf{e}_{1}^{h}+\eta h_{\xi} \partial_{\zeta} \mathbf{e}_{1}^{h}+\left(\mathbf{w}^{h}\right)_{\xi}= & \mathcal{A}_{*}^{h}\left(\eta \mathbf{e}_{1}^{h}+\mathbf{w}^{h}\right)+\left(\mathcal{A}(c)-\mathcal{A}\left(c_{*}\right)\right)\left(\mathbf{q}_{*}^{h}\right) \\
& +\left(\mathcal{A}(c)-\mathcal{A}\left(c_{*}\right)\right)\left(\eta \mathbf{e}_{1}^{h}+\mathbf{w}^{h}\right)+\mathcal{G}^{h}\left(\eta, \mathbf{w}^{h}\right),
\end{aligned}
$$

where

$$
\mathcal{G}^{h}\left(\eta, \mathbf{w}^{h}\right)=\mathcal{F}\left(\mathbf{q}_{*}^{h}+\eta \mathbf{e}_{1}^{h}+\mathbf{w}^{h}\right)-\mathcal{F}\left(\mathbf{q}_{*}^{h}\right)-D_{\mathbf{u}} \mathcal{F}\left(\mathbf{q}_{*}^{h}\right)\left(\eta \mathbf{e}_{1}^{h}+\mathbf{w}^{h}\right),
$$

is smooth on $\mathbb{R} \times\left(\mathrm{id}-P^{h}\right) Y^{1}$ and $\mathcal{G}^{h}(\eta, \mathbf{w})=\mathrm{O}\left(|\eta|^{2}+|\mathbf{w}|_{Y^{1}}^{2}\right)$, uniformly in $h$.

We obtain a first-order system for the three unknowns $h, \eta$, and $\mathbf{w}$ in the same way as in [8], by successively taking the scalar product of (4.6) with $\mathbf{e}_{1}^{\text {ad, } h}$ and $\mathbf{e}_{0}^{\text {ad, } h}$, and then projecting with id $-P^{h}$. The invariance of the $L^{2} \times L^{2}$-scalar product under the $\zeta$-shift $(\cdot)^{h}$ is used to eliminate this shift from each equation. We obtain the equation for $h$,

$$
h_{\xi}=\eta+\mathrm{O}\left(\left|c-c_{*}\right|+|\eta|^{2}+|\mathbf{w}|_{Y^{1}}^{2}\right) \text {, }
$$

which decouples, and the first-order system

$$
\begin{aligned}
\eta_{\xi} & =\mathrm{O}\left(\left|c-c_{*}\right|+|\eta|^{2}+|\mathbf{w}|_{Y^{1}}^{2}\right), \\
\mathbf{w}_{\xi} & =\mathcal{A}_{*} \mathbf{w}+\mathrm{O}\left(\left|c-c_{*}\right|+|\eta|^{2}+|\mathbf{w}|_{Y^{1}}^{2}\right),
\end{aligned}
$$

in which $h_{\xi}$ has been replaced from (4.7). Then by arguing with the center manifold theorem as in [8] we obtain the reduced equation for $\eta$,

$$
\eta_{\xi}=\mathrm{O}\left(\left|c-c_{*}\right|+|\eta|^{2}\right)
$$

and the equation for $h$ becomes

$$
h_{\xi}=\eta+\mathrm{O}\left(\left|c-c_{*}\right|+|\eta|^{2}\right)
$$

We now use a normal form transform by replacing

$$
\widetilde{\eta}=\eta+\mathrm{O}\left(\left|c-c_{*}\right|+|\eta|^{2}\right),
$$

such that $h$ satisfies

$$
h_{\xi}=\widetilde{\eta},
$$


and then $\widetilde{\eta}$ verifies

$$
\widetilde{\eta}_{\xi}=f_{0}(\widetilde{\eta} ; c)=a_{0}\left(c-c_{*}\right)+a_{1} \widetilde{\eta}^{2}+\mathrm{O}\left(\left|c-c_{*}\right|\left(\left|c-c_{*}\right|+|\widetilde{\eta}|\right)+|\widetilde{\eta}|^{3}\right) .
$$

The coefficient $a_{0}$ can be directly computed from the formula

$$
a_{0}\left(c-c_{*}\right)=\left\langle\left(\mathcal{A}(c)-\mathcal{A}\left(c_{*}\right)\right) \mathbf{q}_{*}^{0}, \mathbf{e}_{0}^{\mathrm{ad}}\right\rangle=\frac{2}{\lambda^{\prime \prime}(0)}\left(c-c_{*}\right) .
$$

In order to compute $a_{1}$, we first notice that equilibria of the reduced equation (4.9) correspond to planar traveling waves to $(2.1)$. In particular, any equilibrium $\left(\widetilde{\eta}_{\mathrm{e}} ; c_{\mathrm{e}}\right)$ to $(4.9)$ gives a solution $v_{\mathrm{e}}\left(n_{\mathrm{e}} \cdot(\xi, \zeta)\right)$ to $(4.1)$ with $c=c_{\mathrm{e}}$, where we set

$$
n_{\mathrm{e}}=\left(\sin \left(\psi_{\mathrm{e}}-\varphi_{*}\right), \cos \left(\psi_{\mathrm{e}}-\varphi_{*}\right)\right), \quad \widetilde{\eta}_{\mathrm{e}}=\tan \left(\psi_{\mathrm{e}}-\varphi_{*}\right) .
$$

A straightforward calculation then shows that the profile $v_{\mathrm{e}}$ satisfies the one-dimensional system (2.5) with

$$
n=\left(\sin \psi_{\mathrm{e}}, \cos \psi_{\mathrm{e}}\right), \quad c=c_{\mathrm{e}} \cos \left(\psi_{\mathrm{e}}-\varphi_{*}\right)
$$

Consequently, the planar waves in Lemma 2.4 provide us with the family of equilibria

$$
(\widetilde{\eta} ; c)=\left(\tan \left(\psi-\varphi_{*}\right) ; d(\psi)\right)
$$

in which $d(\psi)$ is the directional dispersion, so that

$$
f_{0}\left(\tan \left(\psi-\varphi_{*}\right) ; d(\psi)\right)=0
$$

for any $\psi$ close to $\varphi_{*}$. Upon comparing the Taylor expansions of $f_{0}$ near $\left(0 ; c_{*}\right)$ and of $(4.11)$ near $\psi=\varphi_{*}$ we find

$$
a_{1}=-\frac{d^{\prime \prime}\left(\varphi_{*}\right)}{\lambda^{\prime \prime}(0)}
$$

Almost planar interfaces are now found as bounded orbits to the reduced equation (4.9). This is a scalar first-order ODE, so that any bounded orbit of the truncated equation

$$
\widetilde{\eta}_{\xi}=\frac{2}{\lambda^{\prime \prime}(0)}\left(\left(c-c_{*}\right)-\frac{d^{\prime \prime}\left(\varphi_{*}\right)}{2} \widetilde{\eta}^{2}\right),
$$

persists to the full equation, and it is either an equilibrium or a heteroclinic orbit. Consequently, nontrivial almost planar interfaces are either interior or exterior corners. A straightforward analysis of this equation then leads to the existence results in the theorem, in the case when the flux is either convex or concave. If the flux $d$ is flat, $d \equiv c_{*}$, all equilibria above have $c=d(\psi)=c_{*}$, so that the reduced equation (4.9) has bounded solutions only if $c=c_{*}$, when the center manifold is filled with equilibria. We then conclude that there are no almost planar interfaces in this case. This completes the proof of the theorem for $c_{\mathrm{g}}=0$. 
Assume now that $c_{\mathrm{g}} \neq 0$. Then the eigenvalue of $\mathcal{A}_{*}$ in the origin is simple with kernel spanned by $\mathbf{e}_{0}$. Therefore, we set

$$
\mathbf{u}=\mathbf{q}_{*}^{h}+\mathbf{w}^{h}, \text { with } P^{h} \mathbf{w}^{h}=P \mathbf{w}=0,
$$

in which $P$ is the spectral projection onto the kernel of $\mathcal{A}_{*}$, and $(\cdot)^{h}$ denotes the $\zeta$-shift as before. We now find the system

$$
\begin{aligned}
h_{\xi} & =\mathrm{O}\left(\left|c-c_{*}\right|+|\mathbf{w}|_{Y^{1}}^{2}\right), \\
\mathbf{w}_{\xi} & =\mathcal{A}_{*} \mathbf{w}+\mathrm{O}\left(\left|c-c_{*}\right|+|\mathbf{w}|_{Y^{1}}^{2}\right) .
\end{aligned}
$$

Since the linear operator $\mathcal{A}_{*}$ is hyperbolic on id $-P$, the equation for $\mathbf{w}$ has no small bounded solutions, except for one equilibrium near $\mathbf{w}=0$, for $c$ close to $c_{*}$. Consequently, the only solutions for which the derivative $h_{\xi}$ stays bounded are those with $h_{\xi} \equiv$ const., and we conclude that there are no almost planar interfaces in this case. This completes the proof of Theorem 1.

\section{$5 \quad$ Examples}

(i)

The simplest example is provided by an anisotropic medium where the diffusion rate depends upon direction,

$$
a^{m}=d^{m} \operatorname{diag}\left(b_{11}, b_{22}\right)
$$

where $b_{11}, b_{22}, d^{m}, m=1 \ldots N$, are positive constants.

A straightforward calculation using the linear dispersion relation gives

$$
c_{\mathrm{g}}=\left(d e_{0}^{\prime}, e_{0}^{\mathrm{ad}}\right)\left(b_{11}-b_{22}\right) \sin (2 \varphi) .
$$

Alternatively, we may rescale $y_{j}=x_{j} / \sqrt{b_{j j}}$ to render the medium isotropic and achieve $\widetilde{c}(\varphi) \equiv c_{\mathrm{o}}$. Transforming back gives the nonlinear dispersion

$$
c(\varphi)=c_{\mathrm{o}} \sqrt{b_{11} \sin ^{2}(\varphi)+b_{22} \cos ^{2}(\varphi)}
$$

from which we find the formula for the group velocity

$$
c_{\mathrm{g}}=\frac{c_{\mathrm{O}}^{2}\left(b_{11}-b_{22}\right) \sin (2 \varphi)}{2 c(\varphi)} .
$$

In particular, this shows that $\left(d e_{0}^{\prime}, e_{0}^{\mathrm{ad}}\right) \equiv c_{\mathrm{o}}^{2} / 2 c(\varphi)$.

For the flux $d$ we obtain

$$
d^{\prime \prime}(\varphi)=\frac{c_{\mathrm{o}}^{4} b_{11} b_{22}}{c(\varphi)^{3}}>0
$$


so that the flux is always convex. Then interior corners exist, provided Hypotheses 2.1 and 2.3 hold, which drift along the interface with nonzero speed, except for the special values of the angle $\varphi=0, \pi / 2$, and the isotropic case $b_{11}=b_{22}$.

(ii)

Another example is provided by viscous conservation laws

$$
u_{t}=\nabla \cdot(a \nabla u)+\nabla \cdot F(u)+c(n \cdot \nabla) u
$$

in which $F=\left(F_{1}, F_{2}\right), F_{j}: \mathbb{R}^{N} \rightarrow \mathbb{R}^{N}$. In this case the traveling-wave equation (2.2) can be integrated once with respect to $\zeta$. We find

$$
(a n \cdot n) u^{\prime}+n \cdot F(u)+c u=\text { const. }
$$

and the condition for existence of shocks

$$
n \cdot\left(F\left(u_{+}\right)-F\left(u_{-}\right)\right)+c\left(u_{+}-u_{-}\right)=0,
$$

where $u_{ \pm}$represent the asymptotic states. This yields the nonlinear dispersion relation

$$
c(\varphi)=c_{1} \sin \varphi+c_{2} \cos \varphi,
$$

in which $c_{1}$ and $c_{2}$ only depend upon $u_{ \pm}$and $F\left(u_{ \pm}\right)$, or

$$
c(\varphi)=c_{*} \cos \left(\varphi-\varphi_{*}\right)+c_{\mathrm{g}} \sin \left(\varphi-\varphi_{*}\right)
$$

It is then straightforward to check that the flux $d$ is flat in the frame moving with the group velocity along the interface, so there are no almost planar interfaces in this case.

We note that here the Fredholm properties required in Hypothesis 2.3 are not satisfied in the usual Sobolev spaces, but they can be achieved by introducing suitable weighted norms.

(iii)

We consider now a perturbed Allen-Cahn equation

$$
u_{t}=\Delta u+u\left(1-u^{2}\right)+c(n \cdot \nabla) u+\varepsilon g(u, \nabla u) .
$$

At $\varepsilon=0$, all planar interfaces are stationary,

$$
u_{*}(x)=\tanh \left(\frac{n \cdot x}{\sqrt{2}}\right),
$$

for $c=0$. It is straightforward to check that the stability assumption, Hypothesis 2.3, holds. We find the linear operators,

$$
\mathcal{L}(k) v=v^{\prime \prime}-k^{2} v+v-3 u_{*}^{2} v,
$$


with spectra contained in $\left(-\infty,-k^{2}-a\right] \cup\left\{-k^{2}\right\}$, for some positive $a$, and linear dispersion relation $\lambda(k)=-k^{2}$. However, since the nonlinear dispersion relation is trivial, $c \equiv 0$, there are no corner defects for $\varepsilon=0$.

For $\varepsilon \neq 0$, we take, for example,

$$
g(\nabla u)=\sum_{n \geq 1} g_{n}\left(\partial_{x_{1}} u\right)^{n}+\sum_{n \geq 1} g_{n}^{\prime}\left(\partial_{x_{2}} u\right)^{n}
$$

in which the coefficients $g_{n}$ and $g_{n}^{\prime}$ satisfy

$$
\limsup _{n \rightarrow \infty}\left(\left|g_{n}\right|^{1 / n}+\left|g_{n}^{\prime}\right|^{1 / n}\right)=M,
$$

for some positive constant $M$, so that $g$ is well defined and smooth in some open ball centered in the origin in $\mathbb{R}^{2}$. More general nonlinearities can be treated in the same way.

Planar interfaces to (5.2) verify

$$
u^{\prime \prime}+u\left(1-u^{2}\right)+\varepsilon g\left(n u^{\prime}\right)+c u^{\prime}=0 .
$$

At $\varepsilon=0$, we have the solution $u=u_{*}, c=0$, above. We then proceed as for (2.5) and solve for $c=c(\varphi, \varepsilon), u=u(\varphi, \varepsilon)$, for small $\varepsilon$. A straightforward calculation then gives the nonlinear dispersion relation

$$
c(\varphi, \varepsilon)=-\varepsilon \frac{\left(g\left(n u_{*}^{\prime}\right), u_{*}^{\prime}\right)}{\left(u_{*}^{\prime}, u_{*}^{\prime}\right)}+\mathrm{O}\left(\varepsilon^{2}\right)=-\frac{\varepsilon}{\alpha_{1}} \sum_{n \geq 1} g_{n} \alpha_{n}(\sin \varphi)^{n}-\frac{\varepsilon}{\alpha_{1}} \sum_{n \geq 1} g_{n}^{\prime} \alpha_{n}(\cos \varphi)^{n}+\mathrm{O}\left(\varepsilon^{2}\right),
$$

in which

$$
\alpha_{n}=\int_{\mathbb{R}}\left(u_{*}^{\prime}(\zeta)\right)^{n+1} \mathrm{~d} \zeta
$$

In particular,

$$
\alpha_{n+1}=\frac{\sqrt{2}(n+1)}{2 n+3} \alpha_{n}, \quad \alpha_{0}=2,
$$

so that the series in (5.4) converge provided $M<\sqrt{2}, M$ being the constant in (5.3). Since the assumptions on stability of planar fronts in Hypothesis 2.3 are verified at $\varepsilon=0$, it is straightforward to check that they hold for small $\varepsilon$, as well. The conditions on the flux $d$ in Theorem 1 are easily obtain from the nonlinear dispersion (5.4), so that for given $g$ one can conclude existence or non-existence of almost planar interfaces, for any sufficiently small $\varepsilon$.

However, we can obtain a more global description if, instead of directly applying the result in Theorem 1 for fixed $\varepsilon$, small, we go back to the proof, and perform the reduction near the planar front $u_{*}$ at $\varepsilon=0$. In this way we can cover a range of asymptotic angles which is independent of $\varepsilon$. In particular, on this larger center manifold we may have, for suitable nonlinear dispersion, more than only two equilibria, for small $\varepsilon$, and consequently more than only one heteroclinic connection. 
We emphasize that it is possible to choose the perturbation $g$ in order to obtain a prescribed nonlinear dispersion relation at order $\varepsilon$. Using Fourier series expansion, we can write

$$
c(\varphi, \varepsilon)=\varepsilon \sum_{n \in \mathbb{Z}} c_{n} \mathrm{e}^{\mathrm{i} n \varphi}+\mathrm{O}\left(\varepsilon^{2}\right)
$$

in which $c_{n} \in \mathbb{C}$, and $c_{n}=\overline{c_{n}}$ for a real dispersion. We then take

$$
g(\nabla u)=\sum_{n \in \mathbb{Z}} g_{n}\left(\partial_{x_{2}} u+\mathrm{i} \partial_{x_{1}} u\right)^{n}
$$

and recover the nonlinear dispersion relation above, at order $\varepsilon$, when $g_{n}=-\alpha_{1} c_{n} / \alpha_{n}$.

In particular, this shows that even though the Allen-Cahn equation possesses no almost planar interfaces, we can, however, find interior and exterior corners by a adding a suitable small perturbation. Moreover, we can prescribe the speed and the asymptotic angles of the defect through the nonlinear dispersion.

\section{$6 \quad$ Steps generated by lateral instabilities}

In this section we replace the stability Hypothesis 2.3 by an instability assumption, and focus on existence of steps. Roughly speaking, we assume that the planar fronts $q(\varphi)$ are stable in one dimension, and that in two-dimensions they stay stable for angles $\varphi<\varphi_{*}$, and become unstable for angles $\varphi>\varphi_{*}$. More precisely, we make the following assumption.

Hypothesis 6.1 (Lateral instability) Assume existence of a family of planar fronts $q(\varphi)$ satisfying (2.5) for angles $\varphi$ close to $\varphi_{*}$. Consider the linearized operators $\mathcal{L}(k ; \varphi)$ defined as in Section 2. We make the following assumptions:

(i) The spectrum of $\mathcal{L}\left(0 ; \varphi_{*}\right)$ is contained in $\{\lambda \in \mathbb{C}: \operatorname{Re} \lambda<0\} \cup\{0\}$.

(ii) The operator $\mathcal{L}\left(0 ; \varphi_{*}\right)$ is Fredholm with index zero, and has a one-dimensional generalized kernel spanned by the derivative of $q\left(\varphi_{*}\right)$.

(iii) The algebraically simple eigenvalue $\lambda=0$ of $\mathcal{L}\left(0 ; \varphi_{*}\right)$ continues to an eigenvalue $\lambda(k ; \varphi)$ to $\mathcal{L}(k ; \varphi)$, for small $k$ and $\varphi$, with

$$
\lambda(0 ; \varphi)=0, \quad \lambda^{\prime}\left(0 ; \varphi_{*}\right)=\lambda^{\prime \prime}\left(0 ; \varphi_{*}\right)=0, \quad \lambda^{\prime \prime \prime}\left(0 ; \varphi_{*}\right) \neq 0, \quad \partial_{\varphi} \lambda^{\prime \prime}\left(0 ; \varphi_{*}\right)>0 .
$$

(iv) The spectrum of $\mathcal{L}(k ; \varphi)$ is contained in $\{\lambda \in \mathbb{C}: \operatorname{Re} \lambda<0\} \cup\{\lambda(k ; \varphi)\}$, for small $k$ and $\varphi$. 
The main result in this section is the following theorem asserting existence of steps along a smooth curve in the parameter-space $(c, \varphi)$.

Theorem 2 (Existence of steps) Assume that Hypothesis 6.1 holds and that the flux $d$ is either convex or concave, $d^{\prime \prime}\left(\varphi_{*}\right) \neq 0$. Then there exists a smooth curve

$$
\varepsilon \mapsto(c(\varepsilon), \varphi(\varepsilon))=\left(c_{*}+\mathrm{O}\left(\varepsilon^{4}\right), \varphi_{*}+\mathrm{O}\left(\varepsilon^{2}\right)\right)
$$

defined for small $\varepsilon>0$, such that the reaction-diffusion system (2.1) possess a family of steady solutions $\left(u_{\varepsilon}\right)_{\varepsilon>0}$ with $\varepsilon$ small which are steps propagating with speed $c(\varepsilon)$ in the direction $n(\varphi(\varepsilon))$.

Remark 6.2 From the proof, it will be clear that the steps are asymptotic to stable planar interfaces. In particular, the essential spectrum of the linearization is marginally stable and can be pushed into the negative left half plane by means of exponential weights. This is in complete analogy with the Korteweg-de Vries equation, which can be formally derived as a modulation equation in the present situation. We conjecture that the steps are actually spectrally stable, that is, the point spectrum of the linearized operator is contained in the closed left half plane. Again, this is suggested by the KdV approximation. Although higher-order terms do not preserve the symmetries of the KdV equation, we suspect that the spectral picture of the $K d V$ equation persists. Indeed, $\Phi=\int u$ is the physically relevant variable of the position of the interface, when u satisfies $K d V$. Linearizing about $\Phi$ only gives a single root of the Evans function in the origin, as opposed to the double root for the linearization at the KdV soliton. This single root is pinned to the origin even when adding perturbations since the derivative of the step in the direction along the asymptotic planar interface provides a localized solution to the linearization at any order.

Proof. [of Theorem 2]

The reduction.

We proceed as in the proof of Theorem 1 in Section 4, and derive first a reduced system describing the corner defects for speeds $c$ close to $c_{*}$ and angles $\varphi$ close to $\varphi_{*}$. In contrast to the proof of Theorem 1, we allow here for nearby directions $n \sim n_{*}$, by taking the angle $\varphi$ as a second parameter.

In the coordinates $(\xi, \zeta)=\left(n_{*}^{\perp} \cdot x, n_{*} \cdot x\right)$ the system $(2.1)$ becomes

$$
\begin{aligned}
\left(a n_{*}^{\perp} \cdot n_{*}^{\perp}\right) u_{\xi \xi} & +\left(\left(a+a^{T}\right) n_{*} \cdot n_{*}^{\perp}\right) u_{\xi \zeta}+\left(a n_{*} \cdot n_{*}\right) u_{\zeta \zeta} \\
& +f\left(u, n_{*} u_{\zeta}+n_{*}^{\perp} u_{\xi}\right)+c \widetilde{n}(\varphi) \cdot\left(u_{\xi}, u_{\zeta}\right)=0,
\end{aligned}
$$


in which

$$
\widetilde{n}(\varphi)=\left(\sin \left(\varphi-\varphi_{*}\right), \cos \left(\varphi-\varphi_{*}\right)\right)
$$

We write again this system as a first order-system

$$
\mathbf{u}_{\xi}=\mathcal{A}(c, \varphi) \mathbf{u}+\mathcal{F}(\mathbf{u})
$$

by taking $\mathbf{u}=(u, v)$, with $v=u_{\xi}$, and then obtain a line of equilibria to (6.2), at $c=c_{*}$, $\varphi=\varphi_{*}$, from the primary planar front $q\left(\varphi_{*}\right)$. Due to the assumptions on the linear dispersion $\lambda(k ; \varphi)$ in Hypothesis 6.1 , the spectrum of the linearized operator $\mathcal{A}_{*}$ is now slightly changed: the eigenvalue $\nu=0$ is algebraically triple, but still geometrically simple. The kernel and generalized kernel are spanned by

$$
\operatorname{ker} \mathcal{A}_{*}=\operatorname{span}\left(\mathbf{e}_{0}\right), \mathbf{e}_{0}=\left(\begin{array}{c}
e_{0} \\
0
\end{array}\right), \quad \operatorname{gker} \mathcal{A}_{*}=\operatorname{span}\left(\mathbf{e}_{0}, \mathbf{e}_{1}, \mathbf{e}_{2}\right),
$$

in which $e_{0}$ is again the derivative of the planar front $q\left(\varphi_{*}\right)$, and $\mathcal{A}_{*} \mathbf{e}_{1}=\mathbf{e}_{0}$ and $\mathcal{A}_{*} \mathbf{e}_{2}=\mathbf{e}_{1}$. We construct the spectral projection $P$ onto the generalized kernel, with the help of the $L^{2} \times L^{2}$-adjoint $\mathcal{A}_{*}^{\text {ad }}$, as in Section 4 , and then the shifted eigenvector $\mathbf{e}_{0}^{h}$, and, analogously, $\mathbf{e}_{1}^{h}, \mathbf{e}_{j}^{\mathrm{ad}, h}, P^{h}, \mathcal{A}_{*}^{\mathrm{ad}, h}$.

We then decompose

$$
\mathbf{u}=\mathbf{q}_{*}^{h}+\eta_{1} \mathbf{e}_{1}^{h}+\eta_{2} \mathbf{e}_{2}^{h}+\mathbf{w}^{h}, \text { with } P^{h} \mathbf{w}^{h}=P \mathbf{w}=0,
$$

in which $q_{*}=q\left(\varphi_{*}\right)$, and $h, \eta_{1}, \eta_{2}$ are real functions depending upon $\xi$. We now follow the proof of Theorem 1, and after the center manifold reduction, and the normal form transformation, we find the system

$$
\begin{aligned}
h_{\xi} & =\eta_{1}, \\
\eta_{1 \xi} & =\eta_{2}, \\
\eta_{2 \xi} & =f_{1}\left(\eta_{1}, \eta_{2} ; c, \varphi\right),
\end{aligned}
$$

in which we have dropped the tilde. We then have to solve the second order ODE

$$
\eta_{1 \xi \xi}=f_{1}\left(\eta_{1}, \eta_{1 \xi} ; c, \varphi\right)
$$

Expanding the reduced system.

We compute now a Taylor expansion for $f_{1}$,

$$
\begin{aligned}
f_{1}\left(\eta_{1}, \eta_{2} ; c, \varphi\right)=\epsilon_{0}( & \left(c-c_{*}\right)+\epsilon_{1} \eta_{1}+\epsilon_{2} \eta_{2}+a_{0}\left(\varphi-\varphi_{*}\right)^{2}+a_{1}\left(\varphi-\varphi_{*}\right) \eta_{1} \\
& \left.+a_{2}\left(\varphi-\varphi_{*}\right) \eta_{2}+a_{3} \eta_{1}^{2}+a_{4} \eta_{1} \eta_{2}\right) \\
+\mathrm{O}( & \left|c-c_{*}\right|\left(\left|c-c_{*}\right|+\left|\varphi-\varphi_{*}\right|+\left|\eta_{1}\right|+\left|\eta_{2}\right|\right) \\
\quad & \left.+\left|\eta_{2}\right|^{2}+\left(\left|\varphi-\varphi_{*}\right|+\left|\eta_{1}\right|\right)^{3}+\left|\eta_{2}\right|\left(\left|\varphi-\varphi_{*}\right|^{2}+\left|\eta_{1}\right|^{2}\right)\right) .
\end{aligned}
$$


As in Section 4, we make use of the family of planar fronts $q(\varphi)$ which provides us here with the following family of equilibria to (6.4):

$$
\left(\eta_{1} ; c, \varphi\right)=\left(\tan \left(\psi-\varphi_{*}\right) ; \frac{c(\psi)}{\cos (\psi-\varphi)}, \varphi\right)
$$

for any angles $\psi$ and $\varphi$ close to $\varphi_{*}$. Inserting these expressions into the Taylor expansion of $f_{1}$, we can recover the expansion of $f_{1}\left(\eta_{1}, 0 ; c, \varphi\right)$ and find

$$
\epsilon_{1}=0, \quad a_{0}=-\frac{c_{*}}{2}, \quad a_{1}=c_{*}, \quad a_{3}=-\frac{d^{\prime \prime}\left(\varphi_{*}\right)}{2} .
$$

In order to compute the remaining coefficients, we use the linear dispersion relation which turns out to determine the stability properties of the equilibria above. We claim that any eigenvalue $\nu$ of the linearization about such an equilibrium satisfies the equality

$$
\lambda\left(-\mathrm{i} \nu \cos \left(\psi-\varphi_{*}\right) ; \psi\right)=c(\psi) \tan (\psi-\varphi) \cos \left(\psi-\varphi_{*}\right) \nu,
$$

in which $\lambda(\cdot, \cdot)$ is the linear dispersion relation in Hypothesis 6.1 (iii). Indeed, to any eigenvalue $\nu$ with $\operatorname{Re} \nu \neq 0$ we can construct stable/unstable manifolds to (6.4) with expansion

$$
\eta_{1}(\xi)=\tan \left(\psi-\varphi_{*}\right)+\bar{\eta}_{1} \mathrm{e}^{\nu \xi}+\mathrm{O}\left(\mathrm{e}^{2 \nu \xi}\right)
$$

for $c=c(\psi) / \cos (\psi-\varphi)$, and $\psi, \varphi$ close to $\varphi_{*}$. These yield solutions to (6.1) of the form

$$
v_{0}(\widetilde{n}(\psi) \cdot(\xi, \zeta))+v_{1}(\widetilde{n}(\psi) \cdot(\xi, \zeta)) \mathrm{e}^{\widetilde{\nu} \widetilde{n}(\psi)^{\perp} \cdot(\xi, \zeta)}+\mathrm{O}\left(\mathrm{e}^{2 \widetilde{\nu} \widetilde{n}(\psi)^{\perp} \cdot(\xi, \zeta)}\right),
$$

in which $\widetilde{\nu}=\nu \cos \left(\psi-\varphi_{*}\right)$. A direct calculation then shows that $v_{1}$ satisfies

$$
\mathcal{L}(-\mathrm{i} \widetilde{\nu} ; \psi) v_{1}=c(\psi) \tan (\psi-\varphi) \widetilde{\nu} v_{1}
$$

This shows (6.5) and proves our claim.

Using (6.5), we can now proceed and compute the remaining coefficients

$$
\epsilon_{0}=-\frac{6}{\mathrm{i} \lambda^{\prime \prime \prime}\left(0 ; \varphi_{*}\right)}, \quad \epsilon_{2}=a_{2}=0, \quad a_{4}=-\frac{\partial_{\varphi} \lambda^{\prime \prime}\left(0 ; \varphi_{*}\right)}{2} .
$$

Summarizing, we have to study the equation

$$
\begin{aligned}
& \eta_{1 \xi \xi}=-\frac{6}{\mathrm{i} \lambda^{\prime \prime \prime}\left(0 ; \varphi_{*}\right)}\left(\left(c-c_{*}\right)-\frac{c_{*}}{2}\left(\varphi-\varphi_{*}\right)^{2}+c_{*}\left(\varphi-\varphi_{*}\right) \eta_{1}\right. \\
&\left.-\frac{d^{\prime \prime}\left(\varphi_{*}\right)}{2} \eta_{1}^{2}-\frac{\partial_{\varphi} \lambda^{\prime \prime}\left(0 ; \varphi_{*}\right)}{2} \eta_{1} \eta_{1 \xi}\right) \\
&+\mathrm{O}\left(\left|c-c_{*}\right|\left(\left|c-c_{*}\right|+\left|\varphi-\varphi_{*}\right|+\left|\eta_{1}\right|+\left|\eta_{1 \xi}\right|\right)\right. \\
&\left.\quad+\left|\eta_{1 \xi}\right|^{2}+\left(\left|\varphi-\varphi_{*}\right|+\left|\eta_{1}\right|\right)^{3}+\left|\eta_{1 \xi}\right|\left(\left|\varphi-\varphi_{*}\right|^{2}+\left|\eta_{1}\right|^{2}\right)\right) .
\end{aligned}
$$


We look for homoclinic solutions to this second order equation. Since $\eta_{1}^{ \pm}=\lim _{\xi \rightarrow \pm \infty} \eta_{1}(\xi)$ corresponds to the asymptotic slope of the corners, homoclinic orbits are precisely step solutions of the reaction-diffusion system.

\section{Scaling and Melnikov analysis}

In order to find homoclinic solution, we first notice that the truncated equation

$$
\eta_{1 \xi \xi}=-\frac{6}{\mathrm{i} \lambda^{\prime \prime \prime}\left(0 ; \varphi_{*}\right)}\left(\left(c-c_{*}\right)-\frac{c_{*}}{2}\left(\varphi-\varphi_{*}\right)^{2}+c_{*}\left(\varphi-\varphi_{*}\right) \eta_{1}-\frac{d^{\prime \prime}\left(\varphi_{*}\right)}{2} \eta_{1}^{2}\right)
$$

does possess homoclinic orbits for $\varphi=\varphi_{*}$, and any $c>c_{*}$ (resp. $c<c_{*}$ ) if the flux is convex (resp. concave). Our task is to show that these orbits persist for the full equation as a family $\left(\eta_{1}^{*}(\varepsilon) ; c(\varepsilon), \varphi(\varepsilon)\right)$, with small $\varepsilon$. As opposed to many systems where the KdV equation provides the leading order approximation, the present system does not possess a reversibility or a Galilei invariance, such that parameters are necessary to show persistence of the homoclinic orbit. Our strategy therefore involves a sequence of transformations and scalings, which isolate a linear damping term $\eta_{1 \xi}$ as the first order correction. Since the damping unfolds the homoclinic transversely, we can then conclude persistence from the implicit function theorem.

First, we eliminate the linear terms in $\eta_{1}$ by setting

$$
\eta_{1}=\bar{\eta}_{1}+\alpha(c, \varphi)
$$

in which $\alpha(c, \varphi)$ is chosen such that

$$
\partial_{\eta_{1}} f_{1}(\alpha(c, \varphi), 0 ; c, \varphi)=0 \text {. }
$$

Since

$$
\partial_{\eta_{1}} f_{1}\left(0,0 ; c_{*}, \varphi_{*}\right)=0, \quad \partial_{\eta_{1} \eta_{1}} f_{1}\left(0,0 ; c_{*}, \varphi_{*}\right)=-d^{\prime \prime}\left(\varphi_{*}\right) \neq 0,
$$

the existence of $\alpha(c, \varphi)$ is insured by the implicit function theorem. In addition, we find the expansion

$$
\alpha(c, \varphi)=\frac{c_{*}}{d^{\prime \prime}\left(\varphi_{*}\right)}\left(\varphi-\varphi_{*}\right)+\mathrm{O}\left(\left|c-c_{*}\right|+\left|\varphi-\varphi_{*}\right|^{2}\right) .
$$

By substituting (6.6) into the reduced system we then find

$$
\begin{aligned}
& \bar{\eta}_{1 \xi \xi}=-\frac{6}{\mathrm{i} \lambda^{\prime \prime \prime}\left(0 ; \varphi_{*}\right)}\left(\left(c-c_{*}\right)+\frac{c_{*}}{2}\left(\frac{c_{*}}{d^{\prime \prime}\left(\varphi_{*}\right)}-1\right)\left(\varphi-\varphi_{*}\right)^{2}-\frac{c_{*} \partial_{\varphi} \lambda^{\prime \prime}\left(0 ; \varphi_{*}\right)}{2 d^{\prime \prime}\left(\varphi_{*}\right)}\left(\varphi-\varphi_{*}\right) \bar{\eta}_{1 \xi}\right. \\
&\left.-\frac{d^{\prime \prime}\left(\varphi_{*}\right)}{2} \bar{\eta}_{1}^{2}-\frac{\partial_{\varphi} \lambda^{\prime \prime}\left(0 ; \varphi_{*}\right)}{2} \bar{\eta}_{1} \bar{\eta}_{1 \xi}\right) \\
&+\mathrm{O}\left(\left|c-c_{*}\right|\left(\left|c-c_{*}\right|+\left|\varphi-\varphi_{*}\right|+\left|\bar{\eta}_{1}\right|^{2}+\left|\bar{\eta}_{1 \xi}\right|\right)\right. \\
&\left.\quad+\left|\bar{\eta}_{1 \xi}\right|^{2}+\left|\varphi-\varphi_{*}\right|^{3}+\left|\bar{\eta}_{1}\right|^{2}\left(\left|\varphi-\varphi_{*}\right|+\left|\bar{\eta}_{1}\right|\right)+\left|\bar{\eta}_{1 \xi}\right|\left(\left|\varphi-\varphi_{*}\right|^{2}+\left|\bar{\eta}_{1}\right|^{2}\right)\right) .
\end{aligned}
$$


Next, we set

$$
\begin{aligned}
& \bar{c}=c-c_{*}+\frac{c_{*}}{2}\left(\frac{c_{*}}{d^{\prime \prime}\left(\varphi_{*}\right)}-1\right)\left(\varphi-\varphi_{*}\right)^{2}+\mathrm{O}\left(\left|c-c_{*}\right|\left(\left|c-c_{*}\right|+\left|\varphi-\varphi_{*}\right|\right)+\left|\varphi-\varphi_{*}\right|^{3}\right) \\
& \bar{\varphi}=\varphi-\varphi_{*},
\end{aligned}
$$

which is a well-defined change of coordinates in a neighborhood of $\left(c_{*}, \varphi_{*}\right)$, so that the equation becomes

$$
\begin{aligned}
\bar{\eta}_{1 \xi \xi}= & -\frac{6}{\mathrm{i} \lambda^{\prime \prime \prime}\left(0 ; \varphi_{*}\right)}\left(\bar{c}-\frac{c_{*} \partial_{\varphi} \lambda^{\prime \prime}\left(0 ; \varphi_{*}\right)}{2 d^{\prime \prime}\left(\varphi_{*}\right)} \bar{\varphi} \bar{\eta}_{1 \xi}-\frac{d^{\prime \prime}\left(\varphi_{*}\right)}{2} \bar{\eta}_{1}^{2}\right) \\
& +\mathrm{O}\left(\left|\bar{\eta}_{1}\right|^{2}\left(|\bar{c}|+|\bar{\varphi}|+\left|\bar{\eta}_{1}\right|\right)+\left|\bar{\eta}_{1 \xi}\right|\left(|\bar{c}|+|\bar{\varphi}|^{2}+\left|\bar{\eta}_{1}\right|+\left|\bar{\eta}_{1 \xi}\right|\right)\right) .
\end{aligned}
$$

We now introduce the scaling

$$
\xi=|\bar{c}|^{-1 / 4} x, \quad \bar{\varphi}=|\bar{c}|^{1 / 4} \widetilde{\varphi}, \quad \bar{\eta}_{1}=|\bar{c}|^{1 / 2} \widetilde{\eta}_{1},
$$

and find the equation

$$
\widetilde{\eta}_{1 x x}=-\frac{6}{\mathrm{i} \lambda^{\prime \prime \prime}\left(0 ; \varphi_{*}\right)}\left(\operatorname{sign}(\bar{c})-\frac{c_{*} \partial_{\varphi} \lambda^{\prime \prime}\left(0 ; \varphi_{*}\right)}{2 d^{\prime \prime}\left(\varphi_{*}\right)} \widetilde{\varphi} \widetilde{\eta}_{1 x}-\frac{d^{\prime \prime}\left(\varphi_{*}\right)}{2} \widetilde{\eta}_{1}^{2}\right)+\mathrm{O}\left(|\bar{c}|^{1 / 4}\right) .
$$

At lowest order, there exists a homoclinic orbit $\widetilde{\eta}_{1}^{*}$, for $\widetilde{\varphi}=0$, and $\operatorname{sign}(\bar{c})=1($ resp. $\operatorname{sign}(\bar{c})=$ -1 ) if the flux is convex (resp. concave). In order to prove persistence, we view the equation as a nonlinear equation

$$
\mathcal{G}\left(\widetilde{\eta}_{1} ; \widetilde{\varphi}, \varepsilon\right)=0
$$

on functions $\widetilde{\eta}_{1} \in C^{2}(\mathbb{R})$, with values in $C^{0}(\mathbb{R})$. Here, $\varepsilon=|\bar{c}|^{1 / 4}$, and we replaced $\bar{c}$ by $\varepsilon^{4}$ and $-\varepsilon^{4}$ for convex and concave flux, respectively. The homoclinic above provides a solution $\mathcal{G}\left(\widetilde{\eta}_{1}^{*} ; 0,0\right)=0$. The linearization

$$
\partial_{\widetilde{\eta}_{1}} \mathcal{G}\left(\widetilde{\eta}_{1}^{*} ; 0,0\right)=\partial_{x x}-\frac{6 d^{\prime \prime}\left(\varphi_{*}\right)}{\mathrm{i} \lambda^{\prime \prime \prime}\left(0 ; \varphi_{*}\right)} \widetilde{\eta}_{1}^{*},
$$

is formally self-adjoint with respect to the $L^{2}$ inner product. The kernels of the linearization and of the (formal) adjoint are both spanned by the derivative $\partial_{x} \widetilde{\eta}_{1}^{*}$. Now, the derivative of $\mathcal{G}$ with respect to the parameter $\widetilde{\varphi}$,

$$
\partial_{\widetilde{\varphi}} \mathcal{G}\left(\widetilde{\eta}_{1}^{*} ; 0,0\right) \sim \partial_{x} \widetilde{\eta}_{1}^{*}
$$

is orthogonal to the range of the linearization, so that we can solve $(6.7)$ for $\left(\widetilde{\eta}_{1}, \widetilde{\varphi}\right)=$ $\left(\widetilde{\eta}_{1}(\varepsilon), \widetilde{\varphi}(\varepsilon)\right)$, and small $\varepsilon$; see also [7] for a similar analysis. This shows persistence of the family of homoclinics and gives the family of steps as claimed in the theorem. 


\section{Discussion}

\section{Convexity of the flux and the nonlinear dispersion curve}

A convenient way to represent wave propagation in anisotropic media is to plot the nonlinear dispersion relation $c=c(\varphi)$, introduced in Section 2, as a curve in the plane. We can plot the vector $c(\varphi) n(\varphi)$ and let $\varphi$ vary, which then traces a curve $\Gamma$ parameterized through

$$
\gamma(\varphi)=c(\varphi)(\sin \varphi, \cos \varphi)=d(\varphi) \cos \left(\varphi-\varphi_{*}\right)(\sin \varphi, \cos \varphi)
$$

We call this curve nonlinear dispersion curve. It turns out that the assumptions on group velocity and flux in Theorem 1 can be read off this curve, and that several properties of corner defects can be detected, as well.

First, the tangent vector to $\Gamma$ at $\gamma\left(\varphi_{*}\right)=c_{*} n_{*}$ is given by $c_{\mathrm{g}} n_{*}+c_{*} n_{*}^{\perp}$. The condition $c_{\mathrm{g}}=0$ therefore is equivalent to a first order tangency between $\Gamma$ and the circle with diameter the vector $c_{*} n_{*}$, or, more generally, any circle with diameter along $n_{*}$ passing through $\gamma\left(\varphi_{*}\right)$.

Next, convexity properties of the flux $d$ can be expressed in terms of the curvature of $\Gamma$,

$$
K(\varphi)=\frac{c^{2}(\varphi)+2 c^{\prime 2}(\varphi)-c(\varphi) c^{\prime \prime}(\varphi)}{\left(c^{2}(\varphi)+c^{\prime 2}(\varphi)\right)^{3 / 2}}
$$

At $\varphi=\varphi_{*}$, we find

$$
K\left(\varphi_{*}\right)=\frac{2}{\left(c_{*}^{2}+c_{\mathrm{g}}^{2}\right)^{1 / 2}}-d^{\prime \prime}\left(\varphi_{*}\right) \frac{c_{*}}{\left(c_{*}^{2}+c_{\mathrm{g}}^{2}\right)^{3 / 2}} .
$$

The first term in the right hand side of this formula,

$$
K_{*}=\frac{2}{\left(c_{*}^{2}+c_{\mathrm{g}}^{2}\right)^{1 / 2}}
$$

represents the curvature of a circle $C_{*}$ whose diameter is given by the length of the tangent vector $c_{\mathrm{g}} n_{*}+c_{*} n_{*}^{\perp}$. It has a first order tangency with the curve $\Gamma$ at $\gamma\left(\varphi_{*}\right)$ when plotted with diameter joining the points $\gamma\left(\varphi_{*}\right)=c_{*} n_{*}$ and $c_{\mathrm{g}} n_{*}^{\perp}$, or, in the explicit parameterization,

$$
\varphi \mapsto\left(c_{*} \cos \left(\varphi-\varphi_{*}\right)+c_{g} \sin \left(\varphi-\varphi_{*}\right)\right)(\sin \varphi, \cos \varphi)
$$

Then we have that the flux

- $d$ is convex if $\Gamma$ lies outside $C_{*}\left(K\left(\varphi_{*}\right)<K_{*}\right)$;

- $d$ is concave if $\Gamma$ lies inside $C_{*}\left(K\left(\varphi_{*}\right)>K_{*}\right)$;

- $d$ flat if $\Gamma$ coincides with $C_{*}$; 

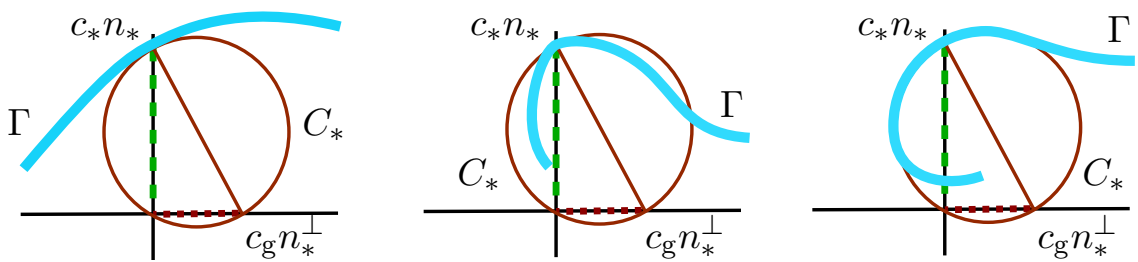

Figure 7.1: Plot of the nonlinear dispersion curve $\Gamma$ and of the circle $C_{*}$ in cases of convex, concave, and flat flux $d$.

for $\varphi$ in a neighborhood of $\varphi_{*}$; see Figure 7.1.

Consider now a speed $c$ and the circle $C$ with diameter the segment connecting $c n_{*}$ with the origin. Then any intersection point of the circle $C$ with the curve $\Gamma$ corresponds to a planar interface moving with speed $c$ in the direction $n_{*}$, since the speed of such an interface in this direction is given by $c(\varphi) / \cos \left(\varphi-\varphi_{*}\right)$. Consequently, the asymptotic planar interfaces to a corner defect propagating in a direction $n_{*}$ with speed $c$, can be found as intersection points of the curve $\Gamma$ with the circle $C$. Furthermore, we can compute the difference between the tangent vectors $t_{\Gamma}$ to the curve $\Gamma$ and $t_{C}$ to $C$ at some intersection point $\gamma_{\mathrm{i}}$, and find

$$
t_{\Gamma}-t_{C}=d^{\prime}\left(\varphi_{\mathrm{i}}\right) \cos \left(\varphi_{\mathrm{i}}-\varphi_{*}\right) n_{\mathrm{i}}
$$

so that

- if $d^{\prime}\left(\varphi_{\mathrm{i}}\right)<0$, the curve $\Gamma$ enters the circle $C$ at $\varphi_{\mathrm{i}}$;

- if $d^{\prime}\left(\varphi_{\mathrm{i}}\right)>0$, the curve $\Gamma$ leaves the circle $C$ at $\varphi_{\mathrm{i}}$.

For the corner defects found in Theorem 1 we always have

$$
d^{\prime}\left(\varphi_{+}\right)<0 \text { and } d^{\prime}\left(\varphi_{-}\right)>0
$$

and we then conclude that

- the corner defect is an interior corner, if the curve $\Gamma$ lies inside the circle $C$ for angles $\varphi$ between the asymptotic angles $\varphi_{+}<\varphi_{-}$;

- the corner defect is an exterior corner, if the curve $\Gamma$ lies outside the circle $C$ for angles $\varphi$ between the asymptotic angles $\varphi_{-}<\varphi_{+}$;

see Figure 7.2. 

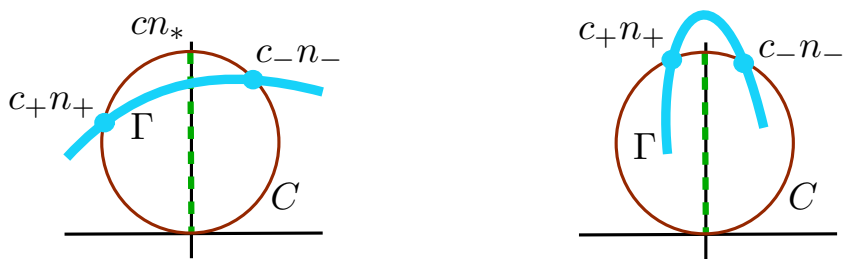

Figure 7.2: Plot of the nonlinear dispersion curve $\Gamma$ and of the circle $C$ in cases of interior (left) and exterior corners (right). The points $c_{ \pm} n_{ \pm}$represent the asymptotic planar interfaces of the defect with $\varphi_{+}<\varphi_{*}<\varphi_{-}$for interior corners (left), and $\varphi_{-}<\varphi_{*}<\varphi_{+}$for exterior corners (right). (The curve $\Gamma$ is oriented clockwise.)

\section{Large corners}

Beyond the (local) bifurcation analysis that we presented above, one can try to predict existence of corners for large angles between the asymptotic interfaces, based on dimension counting arguments for heteroclinic orbits. The small corners that we found are robust saddle-sink connections in a center-manifold. For large corners, such a reduction might not always be possible, still, provided the existence of a corner is guaranteed, one can construct smooth stable and unstable manifolds and count their relative dimensions and codimensions; see [9, 10]. More precisely, let $E_{ \pm}^{\mathrm{cu} / \mathrm{cs}}$ denote the tangent space to the center-(un)stable manifolds of the planar interfaces at $\xi= \pm \infty$, and let

$$
i: E_{-}^{\mathrm{cu}} \times E_{+}^{\mathrm{cs}} \mapsto Y, \quad\left(\mathbf{u}_{-}, \mathbf{u}_{+}\right) \mapsto \mathbf{u}_{-}-\mathbf{u}_{+},
$$

be the injection map. The corner defect will be robust if the intersection is transverse and we will actually find a two-parameter family of corner defects corresponding to varying the asymptotic angles $\varphi_{ \pm}$, if $i$ is onto. Now the $x$ - and $y$-derivatives of the corner defect provide a two-dimensional kernel of $i$ such that the Fredholm index of $i$ needs to be two if $i$ is onto. An analysis analogous to [10] shows that the Fredholm index of $i$ is two if the transport at the asymptotic interfaces, measured by the Cahn-Hoffman vector $c_{ \pm} n_{ \pm}+c_{ \pm}^{\mathrm{g}} n_{ \pm}^{\perp}$, projected on the direction $n_{*}^{\perp}$ parallel to the interface, is directed towards the defect, that is, if

$$
c_{+}\left(n_{+}, n_{*}^{\perp}\right)+c_{+}^{\mathrm{g}}\left(n_{+}^{\perp}, n_{*}^{\perp}\right)<0 \text { and } c_{-}\left(n_{-}, n_{*}^{\perp}\right)+c_{-}^{\mathrm{g}}\left(n_{-}^{\perp}, n_{*}^{\perp}\right)>0,
$$

or equivalently, if

$$
d^{\prime}\left(\varphi_{+}\right)<0 \text { and } d^{\prime}\left(\varphi_{-}\right)>0 .
$$

Corners with these properties correspond to shock-like structures, where small perturbations are transported and annihilated at the center of the corner. Geometrically, these are precisely the conditions on the relative positions of the circle $C$ and the curve $\Gamma$, found for small angles above; see Figure 7.2. 


\section{Open problems}

In the isotropic setting, we showed in [8] how various instabilities give rise to new phenomena. An interesting case is the sideband instability, when $\lambda(k) \sim-k^{4}+\mu k^{2}$, where infinitely many interior and exterior corners, but also steps are created. In the anisotropic setup such an instability would first occur at a specific angle, say $\varphi_{*}$, whereas other angles would still allow for stable front propagation. In particular, we expect such an instability to create a plethora of corner defects with asymptotic interfaces that are asymptotically stable, unlike in the isotropic setting, where all exterior corners and steps are unstable.

A related question is the competition between transversally stable and unstable fronts at a corner defect: suppose a corner is formed between a transversally stable and a transversally unstable planar interface, can the corner be stable in a suitably weighted norm and do these corners provide an alternative to fingering breakup, the typical scenario in transversally unstable interfaces.

\section{References}

[1] M. Bär, A. Hagberg, E. Meron, and U. Thiele. Front propagation and pattern formation in anisotropic bistable media. Phys. Rev. E 62 (2000), 366-374.

[2] G. Bellettini, P.C. Franzone, and M. Paolini. Convergence of front propagation for anisotropic bistable reaction-diffusion equations. Asymptotic Analysis 15 (1997), 325358.

[3] A. Bonnet and F. Hamel. Existence of nonplanar solutions of a simple model of premixed Bunsen flames. SIAM J. Math. Anal. 31 (1999), 80-118.

[4] G. Caginalp and X. Chen. Convergence of the phase field model to its sharp interface limits. European J. Appl. Math. 9 (1998), 417-445.

[5] J.W. Cahn and D.W. Hoffmann. A vector thermodynamics for anisotropic interfaces. 1. Fundamentals and applications to plane surface junctions. Surf. Sci. 31 (1972), 368-388.

[6] J.W. Cahn and D.W. Hoffmann. A vector thermodynamics for anisotropic interfaces. 2. Curved and faceted surfaces. Acta Metall. 22 (1974), 1205-1214.

[7] S.N. Chow and J.K. Hale. Methods of bifurcation theory. Grundlehren der Mathematischen Wissenschaften 251. Springer-Verlag, New York-Berlin, 1982.

[8] M. Haragus and A. Scheel. Corner defects in almost planar interface propagation. Preprint 2003. 
[9] D. Peterhof, B. Sandstede, and A. Scheel. Exponential dichotomies for solitary wave solutions of semilinear elliptic equations on infinite cylinders. J. Diff. Eqns. 140 (1997), 266-308.

[10] B. Sandstede and A. Scheel. Defects in oscillatory media: toward a classification. SIAM J. Appl. Dyn. Syst. 3 (2004), 1-68.

[11] A.T. Winfree. Heart muscle as a reaction-diffusion medium: The roles of electric potential diffusion, activation front curvature, and anisotropy. Int. J. Bif. Chaos 7 (1997), 487-526. 